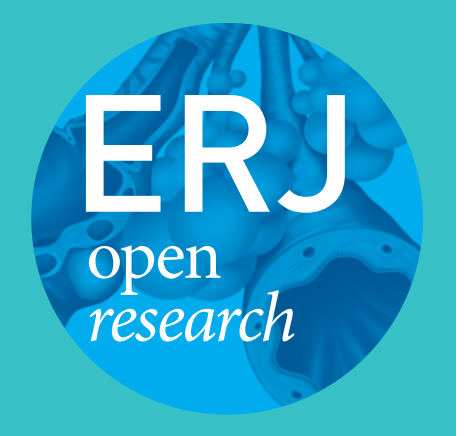

\title{
Acute exacerbation of idiopathic interstitial pneumonias related to chemotherapy for lung cancer: nationwide surveillance in Japan
}

\author{
Yuji Minegishi @1', Akihiko Gemma ${ }^{1}$, Sakae Homma², Kazuma Kishi \\ Arata Azuma (10), Takashi Ogura ${ }^{4}$, Naoki Hamada ${ }^{5}$, Hiroyuki Taniguchi, \\ Noboru Hattori ${ }^{7}$, Yasuhiko Nishioka ${ }^{8}$, Kiminobu Tanizawa (109, Takeshi Johkoh ${ }^{10}$, \\ Takuma Yokoyama ${ }^{11}$, Kazutaka Mori ${ }^{12}$, Yoshio Taguchi ${ }^{13}$, Masahito Ebina ${ }^{14}$, \\ Naohiko Inase ${ }^{15}$, Koichi Hagiwara ${ }^{16}$, Hiroshi Ohnishi ${ }^{17}$, Hiroshi Mukae ${ }^{18}$, \\ Yoshikazu Inoue (19) ${ }^{19}$ Kazuyoshi Kuwano ${ }^{20}$, Hirofumi Chiba ${ }^{21}$, Ken Ohta ${ }^{22}$, \\ Yoshinori Tanino ${ }^{23}$, Fumikazu Sakai ${ }^{24}$ and Yukihiko Sugiyama ${ }^{25}$, for the Diffuse \\ Lung Diseases Research Group
}

\section{ABSTRACT}

Background: Chemotherapy-induced acute exacerbation (AEx) of idiopathic interstitial pneumonias (IIPs) seriously compromises the success of treatment of Japanese lung cancer patients. Here, we conducted a nationwide surveillance to clarify the risk of AEx and compare it with the survival benefit of chemotherapy for this population.

Methods: Advanced nonsmall cell lung cancer (NSCLC) or small cell lung cancer (SCLC) patients with IIPs were retrospectively analysed. For the surveillance of first-line chemotherapy in 2009, we gathered clinical data from 396 patients who received chemotherapy at 19 institutions between January 1990 and July 2009. In a consecutive retrospective study in 2012, we analysed data from 278 patients from 17 institutions who received second-line chemotherapy between April 2002 and March 2012.

Results: Of the 396 patients analysed, 13.1\% developed chemotherapy-related AEx. Combination chemotherapies of carboplatin plus paclitaxel (CP) or carboplatin plus etoposide (CE) were frequently used as first-line treatments. The lowest incidence of AEx was $3.7 \%$ in $\mathrm{CE}$, followed by $8.6 \%$ in $\mathrm{CP}$. In the retrospective study, $16.2 \%$ of the 278 patients developed a second-line chemotherapy-related AEx. The overall response rate by second-line chemotherapy was 7.4\% in NSCLC and $25.7 \%$ in SCLC. The median overall survival from second-line and first-line chemotherapy was 8.0 and 14.3 months in NSCLC, and 8.7 and 16.0 months in SCLC, respectively.

Conclusion: Combination chemotherapies consisting of $\mathrm{CP}$ or $\mathrm{CE}$ are candidates for standard first-line treatments for patients with advanced lung cancer accompanied by IIP. Second-line chemotherapy should be considered for patients remaining fit enough to receive it.

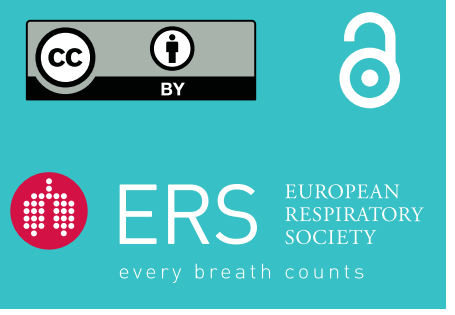

@ERSpublications

The Japanese are at high risk of acute exacerbation of IPF. Therefore, chemotherapy for Japanese lung cancer patients with IIPs is challenging. However, appropriate chemotherapy may give a survival benefit, despite the risk of acute deterioration of IIPs. http://bit.ly/3cROaCy

Cite this article as: Minegishi Y, Gemma A, Homma S, et al. Acute exacerbation of idiopathic interstitial pneumonias related to chemotherapy for lung cancer: nationwide surveillance in Japan. ERJ Open Res 2020; 6: 00184-2019 [https://doi.org/10.1183/23120541.00184-2019].

This article has supplementary material available from openres.ersjournals.com

Received: 25 July 2019 | Accepted after revision: 7 March 2020

Copyright $\odot$ ERS 2020. This article is open access and distributed under the terms of the Creative Commons Attribution Licence 4.0. 


\section{Introduction}

Chronic interstitial lung diseases (ILDs) are one of the most common complications in patients with lung cancer [1-5]. To date, however, there is no consensus regarding optimal treatment strategies for this condition. This is because interventions such as surgical resection, radiotherapy and chemotherapy often trigger fatal deterioration of chronic ILDs, so called acute exacerbation (AEx), in lung cancer patients with ILDs. Pre-existing ILDs are associated with a high risk of acute respiratory failure induced by various anticancer agents. A large cohort study of gefitinib, an epidermal growth factor receptor tyrosine kinase inhibitor (EGFR-TKI), demonstrated that a predisposing background of pre-existing interstitial pneumonias was an independent risk factor for developing acute ILDs; this was true not only in the gefitinib cohort, but also in the control cohort in which patients were treated with conventional chemotherapies [6]. Moreover, AEx of ILDs and severe drug-induced ILDs are more often associated with Japanese populations than with other races. However, the clinical features of AEx triggered by chemotherapy in Japanese lung cancer patients await clarification because only retrospective studies with small sample sizes from single institutions have been performed. Therefore, a large-scale nationwide surveillance for Japanese lung cancer with ILDs is necessary.

At the initiative of the Diffuse Lung Diseases Research Group (DLDRG), organised by the Japanese Ministry of Health, Labour and Welfare, we conducted a preliminary investigation using a simple questionnaire for institutions participating in the DLDRG. This initial surveillance aimed to elucidate the exact incidence of AEx in patients with idiopathic interstitial pneumonias (IIPs) and the trends in the choice of chemotherapy regimen. Individuals with IIPs represent a clinically important subgroup within the spectrum of ILDs. Furthermore, to determine the most appropriate treatment strategy for advanced lung cancer with IIP, we conducted a large-scale multi-institutional retrospective study for patients who received second-line chemotherapy.

\section{Methods and patients}

\section{Study design and patients}

The initial surveillance was requested in July 2009 for institutes participating in the DLDRG. The inclusion criteria were 1) pathologically diagnosed primary lung cancer that was unsuitable for surgery or thoracic radiotherapy because of the disease stage or IIP status; 2) clinically and/or pathologically confirmed IIPs; and 3) chemotherapy history, including molecular targeted therapy. The purposes were to elucidate the incidence of first-line chemotherapy-related AEx of IIP and incidences associated with each regimen in Japanese lung cancer patients. Information was obtained for 396 patients between January 1990 and July 2009 at 19 institutions throughout Japan.

Consecutively, a more detailed large-scale multi-institutional retrospective study that incorporated the results of the initial surveillance was conducted in March 2012. This subsequent retrospective study sourced data from the same institutes, and focused on data from lung cancer patients with IIPs who had received second-line chemotherapy between April 2002 and March 2012. The purpose of this study was to

Affiliations: 'Dept of Pulmonary Medicine and Oncology, Graduate School of Medicine, Nippon Medical School, Tokyo, Japan. ${ }^{2}$ Dept of Respiratory Medicine, Toho University Graduate School of Medicine, Tokyo, Japan. ${ }^{3}$ Dept of Respiratory Medicine, Respiratory Center Toranomon Hospital, Tokyo, Japan. ${ }^{4}$ Dept of Respiratory medicine, Kanagawa Cardiovascular and Respiratory Center, Yokohama, Japan. ${ }^{5}$ Research Institute for Diseases of the Chest, Graduate School of Medical Sciences, Kyushu University, Fukuoka, Japan. ${ }^{6}$ Dept of Respiratory Medicine and Allergy, Tosei General Hospital, Seto, Aichi, Japan. ${ }^{7}$ Dept of Molecular and Internal Medicine Graduate School of Biomedical \& Health Sciences, Hiroshima University, Hiroshima, Japan. ${ }^{8}$ Dept of Respiratory Medicine and Rheumatology, Graduate School of Biomedical Sciences, Tokushima University, Tokushima, Japan. ${ }^{9}$ Dept of Respiratory Medicine, Kyoto University Graduate School of Medicine, Kyoto, Japan. ${ }^{10}$ Dept of Radiology, Kinki Central Hospital of Mutual Aid Association of Public Teachers, Hyogo, Japan. ${ }^{11}$ Dept of Respiratory Medicine, Kyorin University School of Medicine, Tokyo, Japan. ${ }^{12}$ Second Dept of Internal Medicine, Hamamatsu University School of Medicine, Hamamatsu, Japan. ${ }^{13}$ Dept of Respiratory Medicine, Tenri Hospital, Tenri, Japan. ${ }^{14}$ Tohoku Medical and Pharmaceutical University School of Medicine, Dept of Respiratory Medicine, Sendai, Japan. ${ }^{15}$ Tokyo Medical and Dental University, Tokyo, Japan. ${ }^{16}$ Division of Pulmonary Medicine, Jichi Medical University, Shimono, Japan. ${ }^{17}$ Dept of Hematology and Respiratory Medicine, Kochi Medical School, Kochi University, Kochi, Japan. ${ }^{18}$ Dept of Respiratory Medicine, Nagasaki University Graduate School of Biomedical Sciences, Nagasaki, Japan. ${ }^{19}$ Clinical Research Center, National Hospital Organization Kinki-Chuo Chest Medical Center, Osaka, Japan. ${ }^{20}$ Division of Respiratory Diseases, Dept of Internal Medicine, The Jikei University School of Medicine, Tokyo, Japan. ${ }^{21}$ Dept of Respiratory Medicine and Allergology, Sapporo Medical University School of Medicine, Sapporo, Japan. ${ }^{22}$ Japan AntiTuberculosis Association Fukujuji Hospital, Tokyo, Japan. ${ }^{23}$ Dept of Pulmonary Medicine, Fukushima Medical University, Fukushima, Japan. ${ }^{24}$ Dept of Diagnostic Radiology, Saitama International Medical Center, Saitama Medical University, Saitama, Japan. ${ }^{25}$ Nerima-Hikarigaoka Hospital, Tokyo, Japan.

Correspondence: Yuji Minegishi, Dept of Pulmonary Medicine and Oncology, Graduate School of Medicine, Nippon Medical School, 1-1-5, Sendagi, Bunkyo-ku, Tokyo 113-8603, Japan. E-mail: yminegisanms.ac.jp 
elucidate the overall incidence of second-line chemotherapy-related AEx, as well as the incidence associated with each individual treatment regimen; the response rate of second-line chemotherapy and survival time from first- and second-line treatment for each of small cell lung cancer (SCLC) and nonsmall cell lung cancer (NSCLC) were also determined. The medical records of patients were reviewed for age, sex, histology of lung cancer, pathologically or radiologically type of IIP, chemotherapy regimen of each treatment line, chemotherapy-related AEx, the date of start at first- and second-line chemotherapy, outcome, and final survival date. Clinical information for 278 patients at 17 institutions was collated in our department: the data from 180 of these patients were sufficiently rich that a full evaluation, including survival analysis, could be run. The Ethics Committee of the Nippon Medical School approved the study protocol (approval number 28-05-583).

\section{Diagnosis of IIPs}

Based on the combination of clinical and histological findings, diagnosis of IIPs was confirmed in accordance with the criteria proposed by the American Thoracic Society/European Respiratory Society/ Japanese Respiratory Society/Latin American Thoracic Association statement that was published in 2011 [7]. The cases were categorised into two groups based on the type of IIP: usual interstitial pneumonia (UIP) pattern and non-UIP pattern. The UIP pattern consisted of patients with histologically confirmed disease following open-lung biopsy. In the absence of histological evidence, according to the American Thoracic Society/European Respiratory Society/Japanese Respiratory Society/Latin American Thoracic Association criteria, chest high-resolution computed tomography (CT) appearance of definite UIP was defined according to the presence of all four of the following features: 1) subpleural, basal predominance; 2) reticular abnormality; 3) honeycombing with or without traction bronchiectasis; and 4) absence of features listed as "inconsistent with UIP pattern", such as upper or mid-lung predominance, peribronchovascular predominance, extensive ground-glass abnormality, profuse micronodules, discrete cysts, diffuse mosaic attenuation/air trapping, and consolidation in a bronchopulmonary segment or lobe. A non-UIP pattern was classed as any other set of clinical features that did not fall into the categories described above.

\section{Definition of chemotherapy-related AEX of IIPs}

Diagnosis of AEx of IIPs was defined when all of the following criteria were satisfied: 1) an unexplained worsening of dyspnoea within 1 month; 2) evidence of hypoxaemia as defined by worsened or severely impaired gas exchange; 3) new radiographic alveolar infiltrates; and 4) an absence of an alternative explanation, such as infection, pulmonary embolism, pneumothorax, or heart failure. These criteria followed the guideline of IIPs for diagnosis and treatment by the Japanese Respiratory Society in 2004 based on the report of KonDoH and colleagues $[8,9]$. AEx can occur at any point in the course of IIPs; however, based on our previous retrospective study [10], an AEx occurring within 10 weeks of the final chemotherapy treatment was considered as being related to that chemotherapy.

\section{Statistical analysis}

Overall survival was measured as the period from the start of first- and second-line chemotherapy until death by all causes. Median survival time (MST) was analysed using the Kaplan-Meier method. Odds ratios to assess the relative risk of AEx were calculated using logistic regression analysis. We conducted statistical analyses using JMP 11 software (SAS Institute, Gary, NC, USA).

\section{Results}

First-line chemotherapy for lung cancer with IIP

The chemotherapy regimens of first-line chemotherapy and the incidence of chemotherapy-related AEx-IIPs of each regimen are shown in table 1 . The most frequently selected regimen was carboplatin (CBDCA) plus paclitaxel (TXL) (CP) for NSCLC, which was used in 140 patients (35.4\%). Platinum agents plus etoposide (ETP) was the regimen used to treat 120 SCLC patients $(30.1 \%)$. This surveillance revealed that these two regimens are the most widely used first-line chemotherapies for this condition in Japan. Furthermore, these regimens were associated with a lower incidence of AEx-IIPs compared to other regimens. The incidence of AEx was $13.1 \%$ regardless of regimens. The lowest incidence of AEx was $3.7 \%$ in CBDCA plus ETP (CE), followed by $8.6 \%$ in CP and $10.5 \%$ in cisplatin (CDDP) plus ETP (PE) with a low incidence of AEx. Conversely, regimens associated with a relatively high incidence of AEx were CDDP plus vinorelbine (22.2\%), vinorelbine alone (26.7\%), CDDP plus tegafur-uracil (UFT) (29.4\%), CBDCA plus docetaxel (DTX) (66.7\%) and gefitinib (87.3\%). The incidence of AEx-IIPs for each suspected cytotoxic agent is shown in table 2. In combination chemotherapy, it is not possible to determine which agent causes the acute exacerbation, so all administered drugs were considered as potential AEx-inducing agents. The risk of AEx was less in platinum-based combination chemotherapies versus nonplatinum-based agents, and CBDCA was associated with a lower risk of AEx when compared to 
TABLE 1 The incidence of acute exacerbation (AEx) of idiopathic interstitial pneumonias related to each first-line chemotherapy regimen

\begin{tabular}{lcc} 
Regimen & Patients & AEx \\
\hline Carboplatin+paclitaxel & $140(35.4 \%)$ & $12(8.6 \%)$ \\
Carboplatin+etoposide & $82(20.7 \%)$ & $3(3.7 \%)$ \\
Cisplatin+etoposide & $38(9.6 \%)$ & $4(10.5 \%)$ \\
Vinorelbine & $30(7.6 \%)$ & $8(26.7 \%)$ \\
Cisplatin+UFT & $17(4.3 \%)$ & $5(29.4 \%)$ \\
Carboplatin+vinorelbine & $10(2.5 \%)$ & $0(0 \%)$ \\
Cisplatin+vinorelbine & $9(2.3 \%)$ & $2(22.2 \%)$ \\
Docetaxel & $7(1.8 \%)$ & $1(14.3 \%)$ \\
Carboplatin+docetaxel & $6(1.5 \%)$ & $4(66.7 \%)$ \\
Cisplatin+docetaxel & $6(1.5 \%)$ & $1(1.7 \%)$ \\
Gefitinib & $6(1.5 \%)$ & $5(83.3 \%)$ \\
Others & 51 & $10(19.6 \%)$ \\
Total & 396 & $52(13.1 \%)$
\end{tabular}

UFT: tegafur-uracil.

CDDP. Among nonplatinum agents, second-generation agents excluding UFT tended to be associated with a lower risk of AEx than third-generation agents. Moreover, the EGFR-TKI gefitinib was associated with the highest risk of AEx-IIPs.

\section{Patients who underwent second-line chemotherapy}

The characteristics of 278 patients with recurrent lung cancer with IIPs in a multi-institutional retrospective study are summarised in table 3. All patients were Japanese, including 239 males (86\%) with a median age of 69 years (range; 38-85). Of the 249 patients with a specific type of IIPs, 146 patients (58.6\%) exhibited a UIP pattern.

In the trend of second-line chemotherapy, DTX alone was the most frequently selected modality (25.9\%). CP was selected in $11.1 \%$ of patient for both NSCLC and SCLC patients. A wide variety of regimens have been selected as second-line chemotherapies for lung cancer with IIP (table 4). Therefore, there was no bias toward specific regimens in this patient subset.

The incidence of AEX and efficacy by second-line chemotherapy

The incidences of AEx related to second-line chemotherapy and each regimen are summarised in table 4. Regardless of chemotherapy regimen, the incidence of AEx was $16.2 \%$. The incidences of AEx related to DTX, pemetrexed (PEM) and S-1, as standard second-line treatments for NSCLC, were 15.3\%, 33.3\% and

\section{TABLE 2 The association between acute exacerbation (AEx) of idiopathic interstitial} pneumonias and each suspected cytotoxic agent

\begin{tabular}{lcc} 
Agents & Patients & AEx \\
Cisplatin & 91 & $15(16.5 \%)$ \\
Carboplatin & 250 & $20(8.0 \%)$ \\
Etoposide & 124 & $7(5.6 \%)$ \\
Paclitaxel & 146 & $18(12.3 \%)$ \\
Vinorelbine & 51 & $10(19.6 \%)$ \\
Docetaxel & 19 & $6(31.6 \%)$ \\
Gemcitabine & 6 & $1(16.7 \%)$ \\
Vindesine & 17 & $1(5.9 \%)$ \\
UFT & 17 & $5(29.4 \%)$ \\
$\quad$ Platinum-based regimen & 341 & $35(10.3 \%)$ \\
$\quad$ Nonplatinum-based regimen & 49 & $13(26.5 \%)$ \\
\hline UFT: tegafur-uracil. ${ }^{*}$ : gefitinib was excluded. & &
\end{tabular}




\section{TABLE 3 Patient characteristics of the analysis of second-line chemotherapy}

\begin{tabular}{lcc} 
& AEAS $^{\#}$ & SAS $^{\#}$ \\
\hline $\begin{array}{l}\text { Subjects } \\
\text { Age years median (range) }\end{array}$ & 278 & 180 \\
Sex & $69(38-85)$ & $69(38-83)$ \\
$\quad$ Male & $239(86.0 \%)$ & $154(85.6 \%)$ \\
Female & $39(14.0 \%)$ & $26(14.4 \%)$ \\
Histology & & \\
AdC & $101(36.3 \%)$ & $70(38.9 \%)$ \\
SqCC & $70(25.2 \%)$ & $51(28.3 \%)$ \\
AdSqC & $1(0.4 \%)$ & $1(0.6 \%)$ \\
NSCLC (NOS) & $32(11.5 \%)$ & $14(7.8 \%)$ \\
SmCC & $74(26.6 \%)$ & $44(24.4 \%)$ \\
Pattern of IIPs & $146(52.5 \%)$ & $89(49.4 \%)$ \\
UIP & $103(37.1 \%)$ & $66(36.7 \%)$ \\
Non-UIP & $29(10.4 \%)$ & $25(13.9 \%)$ \\
Unclassified & &
\end{tabular}

AEAS: acute exacerbation analysis set; SAS: survival analysis set; AdC: adenocarcinoma; SqCC: squamous cell carcinoma; AdSqC: adenosquamous carcinoma; NSCLC: nonsmall cell lung cancer; NOS: not otherwise specified; SmCC: small cell carcinoma; IIP: idiopathic interstitial pneumonia; UIP: usual interstitial pneumonia. \#: AEAS was evaluated for acute exacerbation and objective response by second-line chemotherapy; SAS consisted of patients for which analysable survival information could be obtained.

$0 \%$, respectively. The incidences of AEx related to nogitecan (TOP) and amrubicin, as standard regimens for SCLC, and CE including re-challenge were $23.1 \%, 33.3 \%$ and $0 \%$, respectively. We infer that CE and $\mathrm{CP}$ tend to be associated with a low risk of AEx in the second-line setting. EGFR-TKIs also engendered a high risk of AEx (44.4\%). No clinical category, including sex, age and pattern of IIPs was significantly correlated with development of chemotherapy-induced AEx.

The objective response rate (ORR) was $7.4 \%$ in the patients with NSCLC, and $25.7 \%$ in the patients with SCLC (table 5). The MST from the first day of treatment to second-line chemotherapy was 8.0 months among 127 patients with NSCLC and 8.7 months among 43 patients with SCLC (figure 1a). MST from the first day of treatment to first-line chemotherapy was 14.3 months among 136 patients with NSCLC and 16.0 months among 44 patients with SCLC (figure $1 \mathrm{~b}$ ). There was no significant difference for characteristics between patients who performed AEx analysis and survival analysis.

TABLE 4 The incidence of acute exacerbation (AEx) of idiopathic interstitial pneumonias related to each second-line chemotherapy regimen

\begin{tabular}{lcc} 
Regimen & Patients & AEx \\
\hline Docetaxel & $72(25.9 \%)$ & $11(15.3 \%)$ \\
Carboplatin+paclitaxel & $31(11.1 \%)$ & $3(9.7 \%)$ \\
Vinorelbine & $24(8.6 \%)$ & $6(25 \%)$ \\
Pemetrexed & $21(7.6 \%)$ & $6(28.6 \%)$ \\
Amrubicin & $18(6.5 \%)$ & $6(33.3 \%)$ \\
S-1 & $14(5.3 \%)$ & $0(0 \%)$ \\
Nogitecan & $13(4.9 \%)$ & $3(23.1 \%)$ \\
Carboplatin+etoposide & $15(5.4 \%)$ & $0(0 \%)$ \\
EGFR-TKls & $9(3.2 \%)$ & $4(44.4 \%)$ \\
Paclitaxel & $7(2.5 \%)$ & $1(14.3 \%)$ \\
Cisplatin+vinorelbine & $6(2.2 \%)$ & $0(0 \%)$ \\
Irinotecan & $6(2.2 \%)$ & $0(0 \%)$ \\
Others & 42 & $5(12.5 \%)$ \\
Total & 278 & $45(16.2 \%)$
\end{tabular}

EGFR-TKI: epidermal growth factor receptor tyrosine kinase inhibitor. 


\begin{tabular}{|c|c|c|}
\hline & NSCLC & SCLC \\
\hline Subjects & 204 & 74 \\
\hline Number of cycles median (range) & $3(1-22)$ & $3(1-6)$ \\
\hline \multicolumn{3}{|l|}{ Response } \\
\hline $\mathrm{CR}$ & 0 & 1 \\
\hline PR & 15 & 18 \\
\hline SD & 68 & 17 \\
\hline PD & 83 & 23 \\
\hline NE & 38 & 15 \\
\hline Overall response rate $n$ & 15 & 19 \\
\hline$\%(95 \% \mathrm{Cl})$ & $7.4(3.8-10.9)$ & $25.7(15.7-38.8)$ \\
\hline Disease control rate $\mathrm{n}$ & 83 & 36 \\
\hline$\%(95 \% \mathrm{CI})$ & $40.7(33.9-49.6)$ & $48.6(34.6-60.9)$ \\
\hline Introduction rate of third-line chemotherapy & $46.6 \%$ & $44.6 \%$ \\
\hline
\end{tabular}

\section{Discussion}

This study is the only large-scale multi-institutional study to analyse the correlation between AEx-IIPs and chemotherapy in advanced lung cancer with IIP. The results of this study have already been published in Japan in 2010, 2012 and 2013. Therefore, our findings have been reflected in clinical practice and have had a strong influence on the design of prospective clinical trials thereafter. Whether chemotherapy for incurable lung cancer patients complicated with ILD is beneficial or harmful has not been elucidated. It is clear that chemotherapy-induced fatal respiratory failure is more frequent in lung cancer patients with chronic ILDs than in those without ILDs. Nevertheless, these patients cannot avoid the risk of AEx without receiving anticancer treatment. Outside of oncology, AEx is also a serious problem in patients who develop chronic ILD. In particular, with Japanese patients with idiopathic pulmonary fibrosis (IPF), AEx manifests frequently during its clinical course and is one of the major causes of mortality [11]. Furthermore, randomised phase II and phase III studies of pirfenidone for IPF in Japan have reported that the incidence of AEx in the placebo groups were $13.9 \%$ over 9 months and $4.8 \%$ over 12 months, respectively $[12,13]$. RichelDi et al. [14] reported that the incidence of adjudicated AEx was $5.4 \%$ and the incidence of investigator-reported AEx in Japanese patients was $12 \%$ in the placebo group during the 52-week observation period of the INPULSIS-1 and -2 nintedanib studies [15]. Additionally, manifestation of AEx with varying frequencies has been confirmed in ILDs other than IPF, such as nonspecific interstitial pneumonia and collagen-vascular-disease-associated interstitial pneumonia $[16,17]$.

The definition of AEx has evolved over time. Therefore, there are slight discrepancies between the international definition and the definition used in this study, because the 2007 definition of AEx proposed by COLLARD et al. [18] does not include hypoxaemia as a required criterion. However, in practice the discrepancies that arise due to these differences are minimal, because almost all patients with AEx had hypoxaemia. An international consensus on the definition of AEx of IPF was established in 2016 [19].

In early 1990, combination regimens containing second-generation cytotoxic agents, such as platinumbased chemotherapy, were already used in clinical practice in Japan. Considering that the older regimens may be safer than regimens containing third-generation cytotoxic agents, we have also included cases who received chemotherapy in the early 1990s in this initial surveillance. Consequently, excluding regimens containing etoposide, only a small number of patients received regimens without third-generation agents. Therefore, we limited our subsequent retrospective study to cases treated after the launch of third-generation drugs and EGFR-TKIs.

Our findings from the initial surveillance show that the risk of AEx is approximately $13 \%$. However, the incidence of fatal acute respiratory failure induced by chemotherapy was markedly higher in lung cancer patients with IIP than in those without ILD. This incidence was similar that reported in past studies (approximately 10\%, range 0-27\%), although the aetiology of ILDs was not taken into account previously. Moreover, our findings showed that choice of treatment regimen has an impact on AEx incidence. In particular, two CBDCA-based regimens, which are part of the TXL and ETP combinations, have been widely used as first-line chemotherapy throughout Japan; our findings suggested that both regimens were less risky when compared to other chemotherapeutic options. The incidences of AEx-ILDs in past studies were $6-12 \%$ in CP-treated patients [20-24] and $12-16 \%$ in patients treated with platinum-based agents 
a)

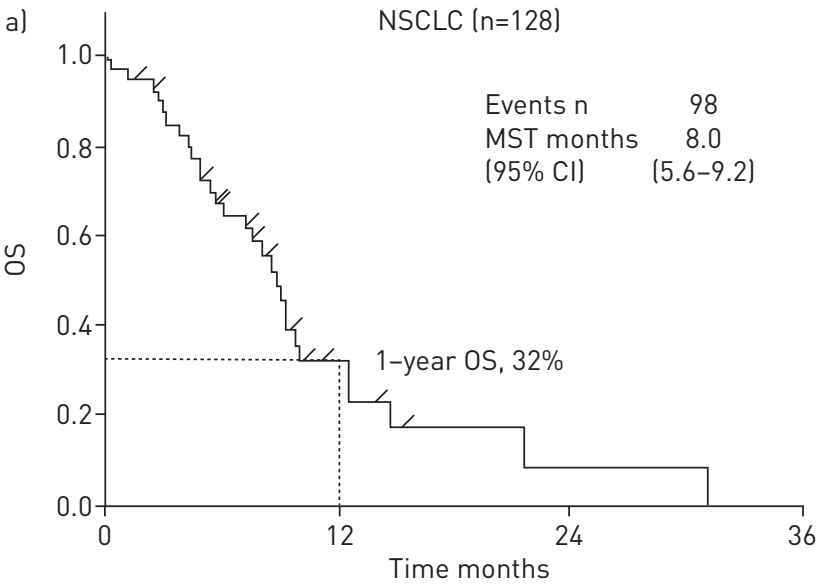

c)

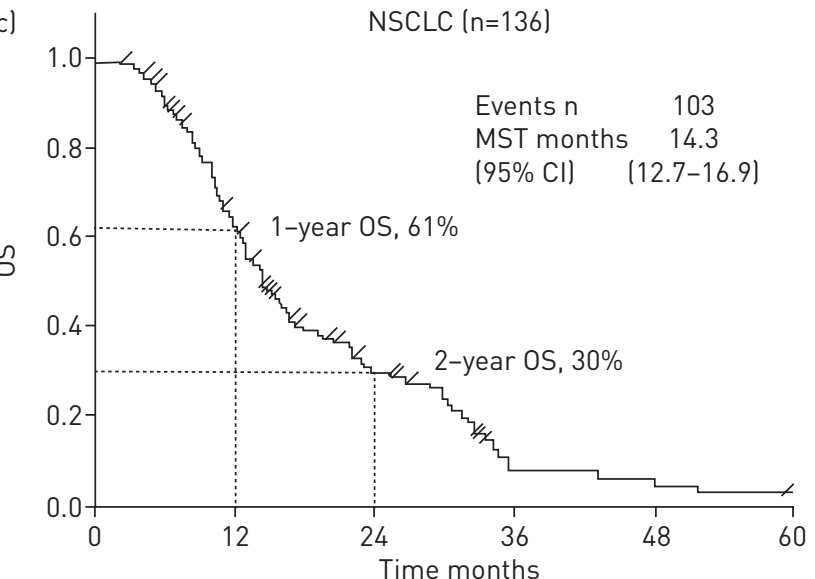

b)

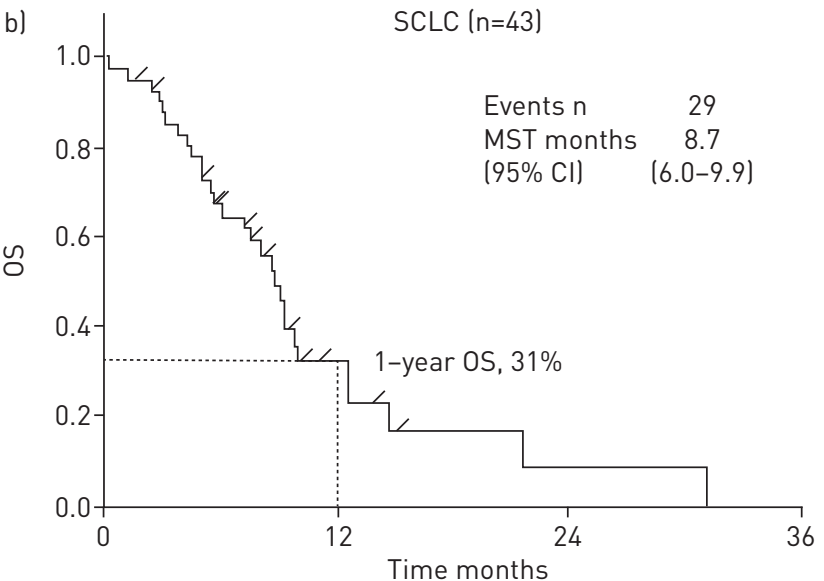

d)

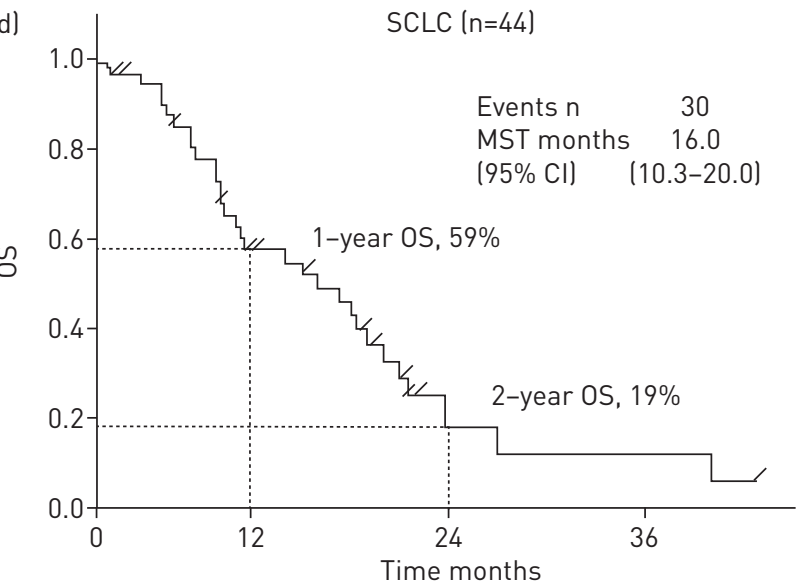

FIGURE 1 Overall survival (OS) a and b) from second-line chemotherapy and c and d) from first-line chemotherapy of a and c) nonsmall cell lung cancer (NSCLC) and b and d) small cell lung cancer (SCLC), respectively. MST: median survival time.

plus ETP [24-27]. These data are similar to our current results ( $8.6 \%$ and 5.8\%, respectively). The initial surveillance was a simple evaluation of a retrospective study. Therefore, differences in patient backgrounds, such as an uneven ratio of UIP versus non-UIP patterns on high-resolution CT (HRCT) scan, could provide potential study limitations. The presence of the UIP pattern on an HRCT scan is a factor associated with susceptibility to AEx-IIPs. However, classification by IIP pattern on an HRCT scan can be complicated due to inconsistencies among radiologists and/or pulmonologists. In this study, the HRCT scan findings of all cases excluding histologically confirmed cases could not be centrally reviewed by the same core group of radiologists. Thus, the IIP pattern in this study reflects the decisions made by individual investigators. Because this compromised the reliability of the IIP pattern, we did not stratify AEx risk according to this characteristic. Nevertheless, this initial surveillance may have revealed the incidence of chemotherapy-induced AEx-IIPs in the community practice setting, and identified the trends of regimen selection in first-line chemotherapy for this study population. Regarding the efficacy of first-line chemotherapy for lung cancer with ILD among previous studies, the ORR and progression-free survival were approximately $40 \%$ (range: $27-61 \%$ ) and about 5 months (range: 3.7-7.2 months) in NSCLC [20-24, 28-32], 70\% (range: 69-88\%) and about 5 months (range: 4.4-5-5 months) in SCLC [24-27], respectively. These results are comparable to those in NSCLC and in SCLC without ILD. The MST is often reported as 7 to 16 months in NSCLC [20-24, 28-32] and from 9 to 10 months in SCLC [25-27]; these MST values are poor compared with the results of large-scale clinical trials for chemotherapy-naïve advanced NSCLC and SCLC. However, the prognosis of those with ILD seemed better than those without this complication who were given best supportive care only. From the perspective of safety and efficacy, we conclude that the risks associated with chemotherapy for this study population are acceptable.

Our findings from the subsequent multi-institutional retrospective study show that the incidence of second-line-chemotherapy-induced AEx was $16.2 \%$. Furthermore, survival analysis revealed that the MSTs from second-line and first-line treatment were 8.6 and 15.7 months for NSCLC patients, and 9.0 and 17.3 months for SCLC patients, respectively. These encouraging results suggest that second-line treatment may 
contribute to improve the prognosis of individuals with this condition. This study shows that DTX, PEM, TOP and amrubicin lead to chemotherapy-induced AEx, and suggests that S-1 alone, which is one of the standard second-line treatments for NSCLC, is associated with a lower risk of AEx-IIPs. Various anticancer agents have been tried in the second-line setting; DTX and PEM are predominant for NSCLC, whereas TOP is more frequently used for SCLC. The incidences of AEx reported in these studies range from $12 \%$ to $22 \%$ [33-37]. Together, our current report and previous studies suggest that second-line chemotherapy should be considered for use in patients who meet the standard second-line chemotherapy criteria for lung cancer patients without IIP and have not experienced acute exacerbation during previous chemotherapy.

\section{Conclusions}

In lung cancer with IIP, the findings of this initial surveillance suggested that combination chemotherapies of platinum agents plus ETP and CP in the first-line setting are associated with an acceptable incidence of AEx-IIPs. We suggest that these combination chemotherapies are candidate standard first-line treatments for advanced or metastatic NSCLC and SCLC with ILD. Moreover, lung cancer patients with IIP who have received second-line chemotherapy have favourable survival rates when compared with those who have received best supportive care only. Furthermore, this retrospective study showed that second-line chemotherapy is associated with ORR and progression-free survival equivalent to those without ILD. Second-line chemotherapy should be considered in lung cancer patients with IIP who remain fit to receive it after the initial chemotherapy. Research to identify risk factors for AEx-IIPs induced by chemotherapy together with prospective studies using specific regimens to evaluate feasibility of first- and second-line chemotherapy are now required to establish the appropriate treatment strategy for this patient population.

Conflict of interest: Y. Minegishi has nothing to disclose. A. Gemma reports grants from the Ministry of Health, Labour and Welfare, Japan, during the conduct of the study; honoraria from Boehringer Ingelheim Japan, Inc., research funding and honoraria from Ono Pharmaceutical Co., Ltd, consulting fees and honoraria from MSD K.K., a subsidiary of Merck \& Co., Inc., and an honorarium from AstraZeneca K.K., outside the submitted work. S. Homma reports research funding and an honorarium from Boehringer Ingelheim Japan, Inc. K. Kishi reports grants and personal fees from Chugai, Ono and Boehringer Ingelheim, grants from Eli Lily, and personal fees from MSD and AstraZeneca, outside the submitted work. A. Azuma reports a consulting fee and an honorarium from Boehringer Ingelheim Japan, Inc., outside the submitted work. T. Ogura reports honoraria from Ono Pharmaceutical Co., Ltd, Taiho Pharmaceutical Co., Ltd, and Boehringer Ingelheim Japan, Inc., outside the submitted work. N. Hamada has research funding and an honorarium from Boehringer Ingelheim Japan, Inc., outside the submitted work. H. Taniguchi reports honoraria from Boehringer Ingelheim Japan, Inc., Asahi Kasei Pharma Corp., AstraZeneca K.K. and Kyorin Pharmaceutical Co., Ltd., outside the submitted work. N. Hattori reports grants from Taiho Pharmaceutical Co., Ltd, Eli Lilly Japan K.K., Daiichi Sankyo Co., Ltd, and Kyowa Hakko Kirin Co., Ltd, outside the submitted work. Y. Nishioka reports grants from the Ministry of Health, Labour and Welfare, Japan, during the conduct of the study; an honorarium from AstraZeneca K.K., and research funds and honoraria from Taiho Pharmaceutical Co., Ltd, Chugai Pharmaceutical Co., Ltd, Eli Lilly Japan K.K., MSD K.K., Ono Pharmaceutical Co., Ltd. and Pfizer K.K., outside the submitted work. K. Tanizawa reports that the Dept of Respiratory Care and Sleep Control Medicine at Kyoto University is funded by endowments from Philips Respironics, Teijin Pharma Ltd., Fukuda Denshi Inc., and Fukuda Lifetec Keiji; and personal fees from Ono Pharmaceutical Co., Ltd, Kyowa Hakko Kirin Co., Ltd, Eizai Co., Ltd, Shionogi \& Co., Ltd, Actelion Pharmaceutical Co., Ltd, MSD, and Boehringer Ingelheim $\mathrm{GmbH}$, outside the submitted work. T. Johkoh has nothing to disclose. T. Yokoyama has nothing to disclose. K. Mori has nothing to disclose. Y. Taguchi has nothing to disclose. M. Ebina has nothing to disclose. N. Inase has nothing to disclose. K. Hagiwara has nothing to disclose. H. Ohnishi reports honoraria from AstraZeneca K.K., Boehringer Ingelheim Japan, Inc., Chugai Pharmaceutical Co., Ltd, Ono Pharmaceutical Co. Ltd, and Taiho Pharma, outside the submitted work. H. Mukae reports grants and lecture honoraia from Daiichi Sankyo, MSD, Sumitomo Dainippon Pharma, Taisho Toyama Pharmaceutical, Astellas Pharma, Boehringer Ingelheim and Shionogi; grants from Taiho Pharmaceutical, Ono Pharmaceutical, Takeda Pharmaceutical, Eli Lilly Japan, Novartis Pharma, Fujifilm Pharma, Meiji Seika Pharma, Toyama Chemical, Eisai and Chugai Pharmaceutical; and lecture honoraria from Pfizer, Kyorin Pharmaceutical and AstraZeneca, outside the submitted work. Y. Inoue reports board member and lecture fees from Boehringer Ingelheim and Shionogi \& Co., Ltd, and research funding from Ono Pharmaceutical Co., outside the submitted work. K. Kuwano reports research funding and honoraria from Boehringer Ingelheim Japan, Inc., and Ono Pharmaceutical Co., Ltd; honoraria from MSD K.K., a subsidiary of Merck \& Co., Inc, AstraZeneca K.K., Pfizer and Daiichisankyo; and research funding from GlaxoSmithKline, outside the submitted work. H. Chiba has nothing to disclose. K. Ohta has nothing to disclose. Y. Tanino reports an honorarium from Boehringer Ingelheim Japan, Inc., and research funding from Eli Lilly Japan K.K., Novartis, MSD K.K. and Shionogi \& Co., Ltd, outside the submitted work. F. Sakai reports personal fees from AstraZeneca, Ono Pharamaceuticals Co. Ltd., Chugai Pharamaceuticals Co. Ltd., Merck Co. Ltd, Rosch Co. Ltd and Boehringer Co. Ltd, outside the submitted work. Y. Sugiyama has nothing to disclose.

Support statement: This study was supported by a grant to the Diffuse Lung Diseases Research Group from the Ministry of Health, Labour and Welfare, Japan.

\section{References}

1 Turner-Warwick M, Lebowitz M, Burrows B, et al. Cryptogenic fibrosing alveolitis and lung cancer. Thorax 1980; 35: 496-499.

2 Hubbard R, Venn A, Lewis S, et al. Lung cancer and cryptogenic fibrosing alveolitis. A population-based cohort study. Am J Respir Crit Care Med 2000; 161: 5-8. 

2009; 14: 723-728.

4 Kawasaki H, Nagai K, Yokose T, et al. Clinicopathological characteristics of surgically resected lung cancer associated with idiopathic pulmonary fibrosis. Surg Oncol 2001; 76: 53-57.

5 Samet JM. Does idiopathic pulmonary fibrosis increase lung cancer risk? Am J Respir Crit Care Med 2000; 161: 1-2.

6 Kudoh S, Kato H, Nishiwaki Y, et al. Interstitial lung disease in Japanese patients with lung cancer: a cohort and nested case-control study. Am J Respir Crit Care Med 2008; 177: 1348-1357.

7 Raghu G, Gollard HR, Egan JJ, et al. An official ATS/ERS/JRS/ALAT statement: idiopathic pulmonary fibrosis: evidence-based guidelines for diagnosis and management. Am J Respir Crit Care Med 2011; 183: 788-824.

8 Kondoh Y, Taniguchi H, Kawabata Y, et al. Acute exacerbation in idiopathic pulmonary fibrosis: analysis of clinical and pathological findings in three cases. Chest 1993; 103: 1808-1812.

9 Akira M, Hamada H, Sakatani M, et al. CT findings during phase of accelerated deterioration in patients with idiopathic pulmonary fibrosis. Am J Roentgenol 1997; 168: 79-83.

10 Minegishi Y, Takenaka K, Mizutani H, et al. Exacerbation of idiopathic interstitial pneumonias associated with lung cancer therapy. Intern Med 2009; 48: 665-672.

11 Natsuizaka M, Chiba H, Kuronuma K, et al. Epidemiologic survey of Japanese patients with idiopathic pulmonary fibrosis and investigation of ethnic difference. Am J Respir Crit Care Med 2014; 190: 773-779.

12 Azuma A, Nukiwa T, Tsuboi E, et al. Double-blind, placebo-controlled trial of pirfenidone in patients with idiopathic pulmonary fibrosis. Am J Respir Crit Care Med 2005; 171: 1040-1047.

13 Taniguchi H, Ebina M, Kondoh Y, et al. Pirfenidone in idiopathic pulmonary fibrosis. Eur Respir J 2010; 35 821-829.

14 Richeldi L, du Bois RM, Raghu G, et al. Efficacy and safety of nintedanib in idiopathic pulmonary fibrosis. $N$ Engl J Med 2014; 370: 2071-2082.

15 Azuma A, Taniguchi $\mathrm{H}$, Inoue $\mathrm{Y}$, et al. Nintedanib in Japanese patients with idiopathic pulmonary fibrosis: a subgroup analysis of the INPULSIS randomized trials. Respirology 2017; 22: 750-757.

16 Park IN, Kim DS, Shim TS, et al. Acute exacerbation of interstitial pneumonia other than idiopathic pulmonary fibrosis. Chest 2007; 132: 214-220.

17 Suda T, Kaida Y, Nakamura Y, et al. Acute exacerbation of interstitial pneumonia associated with collagen vascular diseases. Respir Med 2009; 103: 846-853.

18 Collard HR, Moore BB, Flaherty KR, et al. Acute exacerbations of idiopathic pulmonary fibrosis. Am J Respir Crit Care Med 2007; 176: 636-643.

19 Collard HR, Ryerson CJ, Corte TJ, et al. Acute exacerbation of idiopathic pulmonary fibrosis. An international working group report. Am J Respir Crit Care Med 2016; 194: 265-275.

20 Minegishi Y, Sudoh J, Kuribayashi H, et al. The safety and efficacy of weekly paclitaxel in combination with carboplatin for advanced non-small cell lung cancer with idiopathic interstitial pneumonias. Lung Cancer 2011, 71: 70-74.

21 Shimizu R, Fujimoto D, Kato R, et al. The safety and efficacy of paclitaxel and carboplatin with or without bevacizumab for treating patients with advanced nonsquamous non-small cell lung cancer with interstitial lung disease. Cancer Chemother Pharmacol 2014; 74: 1159-1166.

22 Enomoto Y, Kenmotsu H, Watanabe N, et al. Efficacy and safety of combined carboplatin, paclitaxel, and bevacizumab for patients with advanced non-squamous non-small cell lung cancer with pre-existing interstitial lung disease: a retrospective multi-institutional study. Anticancer Res 2015; 35: 4259-4263.

23 Watanabe N, Taniguchi H, Kondoh Y, et al. Efficacy of chemotherapy for advanced non-small cell lung cancer with idiopathic pulmonary fibrosis. Respiration 2013; 85: 326-331.

24 Kenmotsu H, Naito T, Mori K, et al. Effect of platinum-based chemotherapy for non-small cell lung cancer patients with interstitial lung disease. Cancer Chemother Pharmacol 2015; 75: 521-526.

25 Minegishi Y, Kuribayashi H, Kitamura K, et al. The feasibility study of carboplatin plus etoposide for advance small cell lung cancer with idiopathic interstitial pneumonias. J Thorac Oncol 2011; 6: 801-807.

26 Togashi Y, Masago K, Handa T, et al. Prognostic significance of pre-existing interstitial lung disease in Japanese patients with small-cell lung cancer. Clin Lung Cancer 2012; 13: 304-311.

27 Yoshida T, Yoh K, Goto K, et al. Safety and efficacy of platinum agents plus etoposide for patients with small cell lung cancer with interstitial lung disease. Anticancer Res 2013; 33: 1175-1180.

28 Okuda K, Hirose $\mathrm{T}$, Oki $\mathrm{Y}$, et al. Evaluation of the safety and efficacy of combination chemotherapy with vinorelbine and platinum agents for patients with non-small cell lung cancer with interstitial lung disease. Anticancer Res 2012; 32: 5475-5480.

29 Sekine A, Satoh H, Baba T, et al. Safety and efficacy of S-1 in combination with carboplatin in non-small cell lung cancer patients with interstitial lung disease: a pilot study. Cancer Chemother Pharmacol 2016; 77: $1245-1252$.

30 Watanabe N, Niho S, Kirita K, et al. Vinorelbine and cisplatin in patients with advanced non-small cell lung cancer with interstitial pneumonia. Anticancer Res 2015; 35: 1697-1701.

31 Kakiuchi S, Hanibuchi M, Tezuka T, et al. Analysis of acute exacerbation of interstitial lung disease associated with chemotherapy in patients with lung cancer: a feasibility of S-1. Respir Investig 2017; 55: 145-152.

32 Kanaji N, Tadokoro A, Kita N, et al. Impact of idiopathic pulmonary fibrosis on advanced non-small cell lung cancer survival. J Cancer Res Clin Oncol 2016; 142: 1855-1865.

33 Watanabe N, Niho S, Kirita K, et al. Second-line docetaxel for patients with platinum-refractory advanced non-small cell lung cancer and interstitial pneumonia. Cancer Chemother Pharmacol 2015; 76: 69-74.

34 Kato M, Shukuya T, Takahashi F, et al. Pemetrexed for advanced non-small cell lung cancer patients with interstitial lung disease. BMC Cancer 2014; 14: 508.

35 Fujimoto D, Shimizu R, Kato R, et al. Second-line chemotherapy for patients with small cell lung cancer and interstitial lung disease. Anticancer Res 2015; 35: 6261-6266.

36 Enomoto Y, Inui N, Imokawa S, et al. Safety of topotecan monotherapy for relapsed small cell lung cancer patients with pre-existing interstitial lung disease. Cancer Chemother Pharmacol 2015; 76: 499-505.

37 Suzuki H, Hirashima T, Kobayashi M, et al. Effect of topotecan as second-line chemotherapy for small cell lung cancer patients with interstitial lung disease. J Chemother 2011; 23: 367-370. 